\title{
Aged care: the evolving landscape of research
}

Yih Yiow Sitoh, MBBS, MRCP

S ince the publication of the Report of the Committee on the Problems of the Aged by Singapore's Ministry of Health in $1984,{ }^{(1)}$ the aged care scene in Singapore has progressed by leaps and bounds, with the proliferation of service capabilities as well as different care models, ranging from primary care to tertiary care as well as long-term residential care. ${ }^{(2,3)}$

Alongside these developments in aged care services is a growing body of research regarding different facets of care for older persons within Singapore and its neighbouring countries. This issue of the Singapore Medical Journa/ provides a sampling of such research efforts. The papers published herein include both quantitative and qualitative studies that delve into a wide range of issues including caregiver burden, assessment scales, health-seeking behaviour and end-of-life concerns.

The cross-sectional study by Aman et $\mathrm{al}^{(4)}$ on the impact of caregiving on caregivers adds to the growing body of research examining burdens faced by carers of older persons. Of particular interest is its examination of factors associated with higher burden among carers within a multi-ethnic and multi-cultural population. This should inform the local authorities charged with the task of service planning of how best to support the particular needs of their local populations in the coming years. Ang et al's review ${ }^{(5)}$ of concerns faced by carers of older persons falling at home provides another insight into pressures faced by these caregivers. In a review of 15 publications, the authors explored concerns identified in persons caring for older persons at risk of falls and explicated how these factors impact their physical and psychosocial well-being. This gives another perspective into the burdens of caregivers, which may ultimately guide the development of strategies to help better the lives of older persons and their carers in our communities.

Subramaniam et $\mathrm{al}^{(6)}$ examined the validity of the 12-item World Health Organization Disability Assessment Schedule 2.0 (WHODAS 2.0) on a large sample comprising 2,564 older persons of multi-ethnic backgrounds. The sampling methodology allowed the recruitment of a study population that was representative of the population of older Singaporeans and Singapore permanent residents and, importantly, was structured to accommodate participants of different language proficiencies. The outcomes of this study suggest that the WHODAS 2.0 is an important addition to the armamentarium that health planners and researchers may use in future studies focusing on the prevalence and risk factors for disability among older populations as well as, potentially, the impact of interventions.

Yip et al's article examining advance care planning $(\mathrm{ACP})^{(7)}$ provides an interesting insight into the possibility of including ACP in the comprehensive geriatric assessment for older patients admitted for acute medical problems. This paper adds to the sparse collection of studies on ACP for older persons who are not terminally ill, and highlights factors that support ACP discussions as well as barriers that need to be addressed. Hopefully, this will spur further studies examining the ethical and legal aspects of $\mathrm{ACP}^{(8)}$ within Asian populations - a field that is calling for further exploration.

The study by Lee et $\mathrm{al}^{(9)}$ into the health-seeking behaviour of low-income community-dwelling older persons allows an invaluable opportunity to understand the psyche and perception of this group of vulnerable older persons and, importantly, highlights barriers to care that can be improved upon to optimise health outcomes for this strata of the Singapore population. Meanwhile, $\mathrm{Ng}$ et $\mathrm{al}^{(10)}$ examined the level of physical activity (PA) and sedentary behaviour (SB) in a population of ambulatory older adults attending a primary healthcare clinic in Singapore. This cross-sectional study offers a glimpse into possible modifiable factors affecting PA and SB, such as education and employment opportunities. Addressing some of these factors through social policies may guide interventions to potentially reduce the burden of frailty associated with the growing population of older persons in the coming years.

As evidenced by this selection of articles, aged care research spans a whole gamut of areas, all of which can serve to inform the authorities in terms of priorities in service planning and financing, and ultimately has the potential to improve care for the growing population of older persons.

\section{REFERENCES}

1. Ministry of Culture, Singapore. Report of the Committee on the Problems of the Aged. 1984. Available at: https://www.nas.gov.sg/archivesonline/data/ pdfdoc/755-1984-03-23.pdf. Accessed February 17, 2020.

2. Sitoh YY. Aged care services in Singapore--an overview. Ann Acad Med Singapore 2003; 32:717-22.

3. Sitoh YY. Nursing homes in Singapore: a quiet evolution. Singapore Med J 2003; 44:57-9.

4. Aman Z, Liew SM, Ramdzan SN, Philp I, Khoo EM. The impact of caregiving on caregivers of older persons and its associated factors: a cross-sectional study. Singapore Med J 2020; 61:238-45.

5. Ang SGM, O'Brien AP, Wilson A. Carers' concern for older people falling at home: an integrative review. Singapore Med J 2020; 61:272-80.

6. Subramanian M, Abdin E, Vaingankar JA, et al. Validation of the World Health Organization Disability Assessment Schedule 2.0 among older adults in an Asian country. Singapore Med J 2020; 61:246-53.

7. Yip KF, Wong TH, Alhamid SM, et al. Integrating advance care planning as part of comprehensive geriatric assessment for hospitalised frail elderly patients: findings of a cross-sectional study. Singapore Med J 2020; 61:254-9.

8. Montanari Vergallo G. Advance healthcare directives: binding or informational value? Camb Q Healthc Ethics 2020; 29:98-109.

9. Lee JMG, Chan CQH, Low WC, Lee KH, Low LL. Health-seeking behaviour of the elderly living alone in an urbanised low-income community in Singapore. Singapore Med J 2020; 61:260-5.

10. Ng LP, Koh YLE, Tan NC. Physical activity and sedentary behaviour of ambulatory older adults in a developed Asian community: a cross-sectional study. Singapore Med J 2020; 61:266-71.

Correspondence: Dr Sitoh Yih Yiow, Clinical Services Director, Age-Link Specialist Clinic for Older Persons, 38 Irrawaddy Road, \#06-21, Mount Elizabeth Novena Hospital, Singapore 329563. agelinkclinic@gmail.com 